\title{
CAPÍTULO XIV
}

\section{BIOÉTICA UNIVERSITARIA. UNA PRAXIS DEL QUEHACER INVESTIGATIVO}

\section{Nataliya Barbera de Ramírez}

Doctora en Planificación y Gestión del Desarrollo Regional, Directora del Comité Científico de Alianza de Investigadores Internacionales S.A.S ALININ, Docente Investigadora vinculada al Programa de Arquitectura, Facultad de Humanidades, Arte y Diseño de la Universidad del Sinú, Elías Bechara Zainúm - Colombia. Investigadora Asociada categorizada por MINCIENCIAS. Docente Titular jubilada de pregrado y Postgrado de la UPTAG Venezuela. Directora de tesis de pregrado y postgrado. Ponente y conferencista a nivel nacional e internacionalCorreos electrónicos: nataliaberbera@unisinu.edu.co; nataliyabarbera@gmail.com. ORCID ID: http://orcid.org/0000-0002-45665052

\section{Efraín de Jesús Hernández Buelvas}

Magíster en Biotecnología de la Universidad de Córdoba. Docente investigador vinculado al Departamento Currículum Común Unisinú, Facultad de Ciencias Humanas, Arte y Diseño, Universidad del Sinú, Montería-Colombia. Correo electrónico: efrainhernandezb@unisinu.edu.co. ORCID ID: http://orcid. org/0000-0001-5994-7539

\section{Arney Alfonso Vega Martínez}

Magíster en Estudios Políticos de la Universidad de Caldas. Docente investigador vinculado al Departamento Currículum Común Unisinú y al Programa de Comunicación Social de la Facultad de Ciencias Humanas, Arte y Diseño, Universidad del Sinú, Montería-Colombia. Correo electrónico: arneyvega@ unisinu.edu.co. ORCID ID: http://orcid.org/0000-0001-9627-1662

\section{Nathalie Georgina Ramírez Barbera}

Médico Cirujano, Universidad Nacional Experimental Francisco de Miranda. Especialista en Medicina Interna, Universidad Central de Venezuela. Docente vinculado al Programa de Medicina en el Departamento de Ciencias Básicas de la Salud, Facultad de Ciencias de la Salud de la Universidad del Sinú-Elías Bechara Zainúm. Montería, Colombia. Correo electrónico: nathalieramirez@ unisinu.edu.co.

\section{Nahir Minerva Rovero S}

Doctora en Ciencias Gerenciales de la Universidad Nacional Experimental de las Fuerzas Armadas. Docente de la Universidad Politécnica Territorial Alonso Gamero. Falcón- Venezuela. Correo electrónico:nahrovero@hotmail.com 


\title{
Resumen
}

Reflexionar sobre la bioética universitaria en la praxis del quehacer investigativo, constituyó el objetivo de esta investigación. Se abordó desde una metodología documental en la que se emplearon las técnicas de análisis de contenido y ficha resumen como registro de la información durante la revisión de la literatura. Los resultados indican que la bioética dentro del contexto investigativo genera cambios en el interior de las personas, implica reflexión profunda que promueve la toma de decisiones y renueva la sabiduría humana a través del diálogo, consenso y compromiso con la ciencia y el mundo. Se concluye que la bioética en el contexto de la investigación se asume como una disciplina intrínseca al quehacer científico que garantiza la construcción del conocimiento de y para la vida; por tanto, la bioética ha permitido reconocer, valorar y defender la vida y al mundo desde la ciencia mediante acciones que requieren atención, dedicación y esfuerzo por parte del investigador, como sujeto que trabaja con base en valores y principios éticos que exige la ciencia para lograr un hacer consciente de lo individual y social, conjugado con la preservación del ambiente y de la sociedad.

Palabras clave: Bioética, vida, ambiente, ciencia, investigación.

\section{BIOETTIC PERSPECTIVE OF THE UNIVERSITY MANAGEMENT}

\begin{abstract}
Reflecting on university bioethics in the praxis of what to do was the objective of this research. It was approached from a documentary methodology in which the techniques of content analysis and summary sheet were used as a record of the information during the literature review. The results indicate that bioethics within the research context generates changes within people, implies deep reflection that promotes decision-making and renews human wisdom through dialogue, consensus and commitment to science and the world. It is concluded that bioethics in the context of research is assumed as an intrinsic discipline to scientific work that guarantees the construction of knowledge of and for life; Therefore, bioethics has made it possible to recognize, value and defend life and the world from science through actions that require attention, dedication and effort on the part of the researcher, as a subject that works based on values and ethical principles that science requires to achieve a conscious doing of the individual and social, combined with the preservation of the environment and society.
\end{abstract}

Keywords: Bioethics, life, environment, science, research.

Capítulo resultado de un proyecto de investigación culminado: Gestión pedagógica desde la práctica docente para el éxito estudiantil en la Universidad del Sinú. 


\section{Introducción}

Hoy en día la conciencia del rol ético y ambiental del nuevo hombre en el mundo es un tema de suma importancia y pertinencia que debe asumirse desde la ciencia con el fin de promover los cambios exigidos por la sociedad. Al respecto, conviene pensar en un actuar del ser enmarcado dentro de los valores éticos como lo reporta Rondón, et. al. (2012), los cuales apuntan a consolidar la noción ética del individuo, en donde se venza el egocentrismo y se promueva una visión basada en el altruismo como condición de vida. Por lo tanto, esta esencia del ser humano debe enfocarse en toda la multidimensionalidad del pensar y actuar del investigador universitario, apuntando siempre a mejorar la calidad de vida de la sociedad a la que atiende y su relación armónica con el medio ambiente.

Desde esta perspectiva, se contará con un individuo que no se puede desligar de su esencia de ser humano en su condición de ser viviente, enfrentado a un ambiente del cual se sirve e interactúa, por lo tanto, la bioética conviene ser pensada como núcleo central de la formación profesional de todas las áreas disciplinares, con el fin de lograr una actuación profesional donde se sienta identificado con su misma naturaleza, es decir, como un ente ecobiopsicosocial que obedece a sus rasgos individuales pero que al mismo tiempo atiende lo social.

En este sentido, la bioética es una nueva disciplina denominada bioética global (Maldonado y Gómez, 2013) la misma tiene como base ética la racionalidad humana, revelando un nuevo estado del saber, cuyo fundamento son los conocimientos empíricos de todas las ciencias, es decir, una nueva ética social moderna, orientada hacia la innovación y cambio constante. Asimismo, para el referido autor, la bioética abarca las ciencias de la vida y la sabiduría bajo la ética del medioambiente y las éticas aplicadas, dándole un giro ecológico, en donde se llegue a emplear el conocimiento de cómo usar ese nuevo conocimiento en virtud de lograr la sobrevivencia y mejorar la calidad de vida de la humanidad a nivel del planeta.

En virtud de lo antes expuesto, resulta pertinente fomentar la enseñanza de la bioética en las universidades desde el pregrado y en todos los demás niveles de formación. De esta manera, se garantiza un desempeño apegado a los principios bioéticos de las profesiones; así como también, su aplicabilidad en la praxis investigativa del personal 
que ejerce el quehacer científico desde su área de conocimiento. Centrarse en la bioética del investigador obedece principalmente al rol que estos profesionales desempeñan en la construcción del conocimiento y solución de los problemas del entorno circundante a partir de los productos generados de sus investigaciones.

El investigador universitario es el responsable de hacer ciencia al servicio de la sociedad, por tanto, su quehacer debe estar apegado a las normas y códigos éticos de la profesión, pero a su vez a la ética humana, social y ambiental que exige la realidad global en la actualidad. Es el momento oportuno para actuar desde las éticas integradas o convergentes Holguín, (2019), donde se produzca la unión o religación de la ética individual, social o comunitaria, política, ambiental que permita superar los conflictos y contradicciones éticas entre el bien y el mal que se puedan presentar durante el ejercicio investigativos, lo cual apunta a generar conocimiento científico seguro, verdadero; y, sobre todo, brindar soluciones éticamente apropiadas desde la ciencia.

Por consiguiente, en esta investigación se pretende reflexionar sobre los postulados teóricos de la bioética dentro del contexto universitario y particularmente en el ejercicio de la investigación científica; esto consciente de la necesidad de desarrollar el quehacer científico con estricto apego a la bioética como disciplina, ciencia y/o norma que regula el pensar y actuar del hombre en toda su dimensionalidad.

\section{Fundamentación teórica}

La bioética como disciplina se enfoca en el ser humano y el medio ambiente, considerando la relación armónica entre ambos, en busca de la preservación y mantenimiento de toda clase de vida en el planeta, respaldada por un conjunto de conocimientos y una nueva racionalidad en donde la ética y la vida sean la prioridad. Siguiendo el sentido de lo expuesto la bioética viene a representar una ciencia de límites caracterizada por problemas de frontera (Maldonado, 2015), reconocida por este autor como una disciplina científica debido a que su estudio fundamental es la vida y la ética, en donde la vida misma es un problema de frontera, es decir, un campo accesible al conocimiento. Desde esta concepción, se exige incorporar nuevos saberes que se originan de la bioética y actúan bajo un similar espíritu, una misma expresión y en algunos casos, una plataforma de preguntas, complicaciones y definiciones, implicando de inicio, una visión inter y transdisciplinaria. 
Al explorar la perspectiva teórica de la bioética, se tiene que es un término que involucra etimológicamente dos conceptos ética y bios, por tanto, se concibe como ética de la vida, coincidiendo con lo dicho por Pérez (2008) quien centra a la bioética como un encuentro entre disciplinas basadas en la vida y los valores. De esta manera, se aprecia una conjugación de elementos en donde se privilegia la vida cargada de racionalidad plagada de valores, conducentes a resguardar al planeta y a sus integrantes.

Por su parte, García (2010) afirma que la bioética es una ética aplicada a la vida del ser humano que evoluciona de manera progresiva en correspondencia con el desarrollo del hombre, aunado a ello, involucra no solo al ser humano sino también su relación con el ambiente, cuyas acciones están regidas por valores basados en la racionalidad humana y ambiental apuntan a un actuar ético del individuo en todas sus facetas: personal, social, profesional, investigativa, entre otras.

Por otro lado, Schimidt (2007) orienta su definición de bioética desde una visión interdisciplinaria cuyo punto de encuentro es el hombre consigo mismo y con su entorno, entendiendo por entorno a las otras personas y al ambiente. El autor no se circunscribe a una mera visión de la vida sino pretende darle al ser humano a través de la bioética un enfoque global de su calidad de vida, el conocimiento científico, el ambiente y su hábitat ofreciéndole la posibilidad de constituir un mundo lleno de posibilidades al integrar su yo interno con la propia vida, su salud y las ciencias. Lo anterior, le otorga significado a la bioética como aquella en donde todas las ciencias se unen con el pensamiento filosófico en pro de lograr encausar acciones hacia la vida humana y el medio en donde interactúa.

Ahondando en el término bioética muestra una visión compleja, reflejada por Mazzanti (2010) al definirla desde una perspectiva integral, involucrando vida y ser humano con el fin de apropiarse de diferentes puntos de vista éticos y darle solución a los conflictos o incertidumbres presentes en la vida del ser humano. En consecuencia, se puede afirmar la visión general dada al término bioética y su sentido humanista ante un mundo caótico y lleno de incertidumbres, el hombre debe mirar más allá de su zona de comodidad para incorporar la ética como filosofía de pensamiento y, por ende, de sus acciones. Estos planteamientos conducen a asumir que el hombre debe saber enfrentarse 
a los desafíos y lograr resolverlos de una forma tal que sume beneficios y bienestar para él y su entorno.

Dentro de este orden de ideas conviene distinguir a Martínez (2011), quien expone que la bioética es un concepto extenso en su concepción, debido a la incorporación de la perspectiva de filosofía moral, es decir, como forma lícita de interesarse en la vida humana, por lo tanto, el autor define a la bioética basada en elementos interdisciplinares, capaz de estudiar y resolver los problemas creados por el mismo hombre y su efecto en la colectividad a través de cuatro áreas principales: teórica, práctica, reglamentaria y cultural. Llegado a este punto, se considera a la bioética como un conjunto de ciencias interdisciplinarias e interdependientes quienes velan por crear puentes entre el ser humano y el ambiente; entre el profesional y la ciencia, pues es a partir de la actuación bioética del investigador que resulta posible solucionar conflictos del entorno desde el conocimiento científico generado mediante la investigación.

De acuerdo con las reflexiones de Martínez (2011) y Maldonado (2005), la bioética no solo interconecta el sentido individual del ser humano sino un punto de vista más complejo donde la mente humana reflexiona, se hace viva frente a sus problemas y los del entorno dándole una perspectiva más racional y filosófica a su existencia aquí en la tierra, emanada de una objetividad compartida con un mundo del cual es participe e integrante a la vez. Por lo tanto, la realidad tiende a ser cambiante, plagada de emergencias, en donde el hombre debe ser capaz de tener las herramientas necesarias para transformar la realidad, por lo tanto, cobra fuerza la perspectiva bioética que posee el investigador como ente promotor de la ciencia, el conocimiento y generador de soluciones transformacionales.

De manera similar, la bioética es una disciplina científica con indudables y reales expectaciones éticas; además, como una ciencia de frontera se concibe y desenvuelve tomando en cuenta los conflictos propios de las demarcaciones del conocimiento; por consiguiente, representa un semillero de conocimiento accesible a todos los límites de la ciencia. En este mismo orden de ideas, Martin (2014) afirma que la bioética viene a ser una "inteligencia integradora de vida, plataforma de capacidades para elegir horizontes, transformar y construir posibilidades para la vida" (p.60). Es decir, una 
forma de razonamiento intrínseca del ser humano dirigido a mejorar su calidad de vida y su interrelación con el ambiente, aunado a un conjunto de potencialidades manejadas de forma prospectiva, en donde los valores para la vida y lo ecológico sean elementos preponderantes.

\section{Metodología}

La orientación metodológica desarrollada en esta investigación fue asumida desde la racionalidad deductiva, con el fin de reflexionar sobre la bioética universitaria desde el quehacer investigativo a partir de los resultados derivados del razonamiento lógico. Consciente de que a través del análisis del texto escrito se accede a situaciones, experiencias, actividades y conocimientos diversos, se empleó la investigación bibliográfica o documental, la cual consistió en la revisión exhaustiva del material bibliográfico existente con respecto a la bioética como fenómeno de estudio y poder identificar, seleccionar y organizar la información del documento escrito con la finalidad de dar cuenta según las categorías de análisis de la investigación (Martínez, 2011). Para ello, se empleó la técnica de análisis de contenido lo que permitió extraer las perspectivas y constructos teóricos más relevantes de los textos, artículos y capítulos de libros que versan sobre la temática estudiada y que se reportan en la bibliografía.

\section{Resultados}

Los fundamentos teóricos esbozados anteriormente reflejan el carácter humano de la bioética y cómo ha sido transformada su visión, de una ética asociada a la deontología y a la medicina, con el fin de lograr articular una nueva forma de ver el mundo que rodea al ser humano y darle un sentido más sublime a su existencia. Así mismo, Junges (2008) rebasa el quiebre con la moral común, al plantear que las obligaciones del individuo están constituidas por los contenidos e influencias de los conocimientos recientes relacionados a la vida, la salud y el ambiente; igualmente, para Caicedo (2020) "la moral es entendida como una especie de Constitución Política interna que se tiene que trabajar a diario" (p.89). Implica obligaciones y contenidos que son puestos en práctica por el investigador durante su ejercicio científico y su mundo cotidiano.

Al revisar la historia de la bioética, conduce a considerar lo expuesto por Escobar y Escobar (2010), quienes marcan la génesis de la bioética y sus principales corrientes. En 
este sentido para Platón, la meditación filosófica se dirige a la vida y como es menester vivirla, solo se puede vivir de forma virtuosa, es decir basada en el bien. En este mismo orden de ideas Aristóteles, (384-322 AC), concibe que las virtudes del ser humano también son la base para una vida llena y va a obedecer a la esencia del hombre. Desde esta visión filosófica de la antigüedad puede apreciarse que la concepción ética posee el carácter de aplicabilidad de las virtudes humanas a la vida; esto implica, transferibilidad a su quehacer y, en este ámbito, al ejercicio de la investigación científica.

En una perspectiva más medieval, se parte de que Jesús indica que el amor incondicional representa la relación perfecta para perfeccionar el componente humano en donde la bondad es el espejo de su interior, sabiendo que el hombre bueno expone cosas buenas en sus acciones y del malo solo saca cosas malas, en concordancia con lo anterior Tomas de Aquino propone, que todo debe ser armónico en el hombre, su razón y su acción; el hombre hace el bien porque es lo razonable y cuando hace el mal está fuera del equilibrio de la razón. Interesante reflexión que conduce a pensar la bioética desde la armonía del ser con base a la razón o logicidad al pensar y actuar, condiciones esenciales para la práctica científica.

En contraposición con lo anterior, se encuentra el pragmatismo, este enfoque le da libertad al pensamiento de pensar, opinar, señalar, ya que se enfrenta y entiende que existen variedad de puntos de vista, apuntando exclusivamente a los resultados de las decisiones tomadas, sin considerar las diatribas de ideas sobre la decisión. Siguiendo estas corrientes, se encuentran las éticas formales esbozadas por Kant, quien plantea su filosofía en las formas y se manifiestan en tipos formales de hechos ejecutados por el hombre que son correctos o incorrectos, luego se presenta el utilitarismo, quienes esbozan que para ellos son buenas esas cosas que se hacen y causan prosperidad.

Asimismo, exponen que el utilitarismo ayuda a beneficiar a un individuo, a su entorno familiar y a la sociedad; el utilitarismo no es individualismo, se dirige hacia acciones perfiladas a la generosidad, por lo que conviene destacar que, este carácter de generosidad se convierte en un valor fundamental para la ciencia, donde el quehacer científico apunta a la resolución de problemas sociales, ambientales, políticos, técnicos, tecnológicos, entre otros. 
De igual manera, Escobar y Escobar (2010) refieren que la bioética utilitarista tiene como principio básico hacer el bien a un mayor número de personas, direccionado por valores individuales, los cuales se apoyan en los valores de la sociedad. Enseguida surge la bioética universalista, cuyas disposiciones conviene tomar en cuenta el sentir de la colectividad de los individuos implicados en la disyuntiva ético. De la misma forma, ostenta la bioética personalista, en donde el punto central de toda la discusión es la persona y su condición de ser facultada sobre los beneficios de las demás personas; así como, de organismos y sociedades, por lo tanto, allí está el bien real de la persona.

Atendiendo al discurso de los autores, incluyen tres principios guías de la bioética, los cuales se proyectan a continuación: El principio de beneficencia, orientado a brindar un bien al beneficiario; el principio de autonomía asociado a la autoridad de regirse a uno mismo y capacidad de tomar medidas o acciones con respecto a nuestro propio cuerpo $\mathrm{y}$; finalmente, el principio de no maleficencia, direccionado al no ocasionar un perjuicio de modo deliberado. Considerando los principios antes señalados, puede decirse que la investigación científica dentro del ámbito universitario debe apuntar a brindar un bien a otro, cuyos resultados están basados en la toma de decisiones acordes a los principios individuales y sociales; por último, no generar perjuicios ni daños. Al respecto, Caicedo (2020), refiere que el "Hacer el bien va más allá del beneficio individual, puesto que el sujeto tiene que contribuir a toda la comunidad. Quien ejecuta buenas acciones es una persona que seguramente está más cerca del conocimiento" (p.89).

Al considerar los planteamientos de Martin (2014), Martínez (2011) y Maldonado (2005), puede decirse que la bioética viene a personificar un diálogo interdisciplinario entre ética y vida, de esta forma, la bioética trabaja bajo los principios morales de la conducta humana en el campo de las ciencias de la vida, apuntando hacia distintas disciplinas, desde las médicas hasta las humanistas, económicas, filosóficas, políticas y también el derecho. Esto es indicativo de que la ética es parte importante de la bioética, otorga la connotación de ética de la vida, al integrar varias disciplinas en pro de relacionar al ser humano con su ambiente y en la búsqueda del bienestar colectivo.

Por otra parte, Barbera et al. (2019), destacan que la bioética es ciencia de los valores que forman parte de la esencia humana, cuya actuación apunta a la búsqueda de la 
calidad de vida de los ciudadanos, mediante la integración del individuo con el ambiente y su subsistencia, por lo tanto, la bioética persigue el equilibrio armónico y perfecto de cuerpo, mente y espíritu. En atención a lo expuesto, la bioética genera cambios en el interior de las personas, implica reflexión profunda tendiente a promover la toma de decisiones y acciones, a renovar la sabiduría humana a través del diálogo, consenso y compromiso, a agrupar la sabiduría individual y social en todo el quehacer cotidiano del ser, de allí su importancia en la actividad investigativa de las universidades.

Consciente de que la labor científica exige un apego a los principios y valores éticos requeridos para la construcción de conocimiento, conviene pensar en la ética de la vida o bioética para desarrollar las investigaciones enmarcadas en el equilibrio armónico del ser, al integrar cuerpo, mente, espíritu en el quehacer reflexivo del investigador Islas, y Cortés. (2020). De esta manera, el actuar investigativo desde la bioética involucra las ciencias de la naturaleza, pero al mismo tiempo, se apoya en otras ciencias para configurarse como ciencia inter y transdisciplinaria con fuertes componentes éticos, direccionados hacia el mejoramiento de la calidad de vida y la preservación del ambiente.

Por consiguiente, la bioética desde el quehacer científico se basa en la sabiduría individual y social para ofrecer el cuidado de la vida como un trabajo donde se crea el mundo civilizado y al conocimiento, involucrando lo ético y lo científico con miras a preservar la vida humana y la del planeta, en donde juega un papel fundamental la sociedad actual y su preocupación por el cuidado del ser humano. Al respecto, Velasco (2009) describe la bioética como un objetivo para la gestión, mediante la forma de cómo emplear ese conocimiento para el logro de la prosperidad de la sociedad, siendo su estudio central la supervivencia de la humanidad; enfatizando en la mejora de la calidad de vida, uniendo el bios y el ethos con el fin de fomentar un nuevo conocimiento a partir de lo biológico y lo humano.

Desde esta perspectiva, la sociedad actual requiere una nueva forma de emplear conocimientos para ser enfocados en el hombre, su calidad de vida, la preservación de la vida como tal en el planeta, esa conservación parte de la biología como ciencia para incluir otras ciencias como las sociales y las humanidades con mayor relevancia en la conciencia. De lo anterior se consolida la bioética como una ciencia destinada a 
la supervivencia humana, pero con el consentimiento e integración de otras ciencias: biología, sociales y humanas bajo preceptos de la filosofía.

Estas reflexiones conducen a comprender que la bioética es un nuevo tipo de racionalidad humana, en donde lo ético y científico se unen para tener en cuenta el cuidado de la vida, mediante un compendio de fundamentos teóricos y prácticos, dirigidos a poder enlazar muchas disciplinas al servicio del individuo y su felicidad. Por lo tanto, el perfeccionamiento de una nueva ciencia basada en el hombre y dirigida hacia el hombre, se ocupa de la ética y la vida, en la racionalidad humana, en configuraciones futuras, en gestiones correctivas y preventivas destinadas a mermar los deterioros originados por las labores humanas.

En consecuencia, la bioética viene a representar una nueva forma de disciplina científica, la cual traspasa sus límites y se convierte en una ciencia de frontera. En este sentido, no solo se ocupa del bios y la ética, sino que integra sus epistemes, dirigiéndose a atender los diversos problemas del hombre, proporcionándole un carácter inter y transdisciplinario en la generación de conocimiento donde convergen infinidad de conocimientos bajo la tutela de la ética y los postulados de la biología, con el fin de atender los problemas originados de la acción humana desde la participación de otras ciencias en un todo interrelacionado y hologramático brindando nuevas perspectivas para lograr la supervivencia humana y por ende de las generaciones futuras.

\section{Conclusiones}

De las reflexiones anteriores, se reconoce que la bioética en el contexto de la investigación científica se asume como una disciplina intrínseca al quehacer investigativo para garantizar la construcción del conocimiento de y para la vida, atendiendo a los problemas humanos, ambientales por vía inter y transdisciplinaria. En este sentido, es responsabilidad del investigador actuar con base en los valores y principios éticos que exige la ciencia, un hacer consciente de lo individual y social, conjugado con la preservación del ambiente y la vida para que puedan ser reconocidos por la sociedad como sujetos que trabajan por un mejor porvenir.

Desde esta perspectiva, la bioética es ciencia y arte representada en valores que se cimientan en la esencia humana y en espacios de acción direccionados a la búsqueda de 
la calidad de vida; se asume de una forma integral orientada a conducir y darle orden a los distintos procesos del quehacer científico; enfocada en lo humano y su relación con el ambiente donde la racionalidad en el pensar y actuar son el componente fundamental en la producción y generación de conocimiento. De allí que, el carácter humano se une a lo científico y viceversa, creando una dialéctica entre la bioética y la ciencia, bajo una premisa consiente y reflexiva de la práctica investigativa, en donde las funciones del investigador van de la mano con lo ambiental o ecológico; esto indica que hacer ciencia desde los principios bioéticos apunta al desarrollo científico desde una visión humana (individual), social (colectiva) y ecológica (ambiental) constituyéndose en el motor preponderante en fomentar una nueva conciencia científica con la finalidad de resguardar al ser humano y su ecosistema.

En este sentido, la bioética aporta un viraje en el hacer científico cuyo núcleo es un sujeto investigador consciente de que sus acciones dañan al otro, para ello, su conocimiento cultural está presente en el obrar investigativo permitiéndole hacer ciencia desde una posición en la que pueda transformar las estructuras de su campo social y ambiental, resaltando la importancia de ese mundo o lugar en el que los humanos se han desarrollado y gira su dinámica relacional. Por consiguiente, la bioética ha permitido reconocer, valorar y defender la vida y el mundo desde la ciencia mediante acciones que requieren atención, dedicación y esfuerzo por parte del investigador. Esta labor parte por atender las necesidades del otro, sus intereses y expectativas; dedicar tiempo, recursos y herramientas metodológicas para trazar rutas o caminos que obedezcan a la incertidumbre o comportamiento complejo de la realidad o del ecosistema cambiante que se vive actualmente; y esfuerzo en las acciones que den sentido al transitar humano por el mundo.

Finalmente, no es posible entender la bioética sino se establece una constante reflexión del accionar del investigador con el fin de lograr sintonizar lo pensado y hablado en torno al mundo circundante, creando la interdependencia y relación dialéctica entre bioética - vida - mundo como una forma de emprender los cambios exigidos por la sociedad garantizando la preservación de la vida y del ecosistema. En este sentido, al prestar atención a esta interrelación se visualiza la importancia de la bioética en el quehacer científico, cuyas acciones buscan la conservación del medio ambiente y el 
mejoramiento de la calidad de vida; por ende, la bioética constituye la esperanza de vida en el planeta tierra.

\section{Referencias bibliográficas}

Barbera, N. Flórez, E. Hernández, E. Vega, A. y Chirinos, Y. (2019. Bioética Gerencial. Nueva Forma de Dirigir las Organizaciones Universitarias. En Y. Chirinos, A. Ramírez, R. Godínez. N. Barbera y D. Rojas. (Coords.), Tendencias en la Investigación Universitaria: Una visión desde Latinoamérica Vol. VII... (pp. 211-224). Venezuela: Fondo Editorial Universitario Servando Garcés de la Universidad Politécnica Territorial Alonso Gamero. www.doi.org/10.47212/ Tendencias_vii 2019 15

Caicedo. J. (2020). La ética y la moral en el ámbito educativo y cultural. Una perspectiva a partir de Alfonso Reyes. En Garzón-Pascagaza, É. J., Montoya-Montoya, L. G., Muñoz-Buitrago, D. A., García, H., Hernández-Álvarez, J. F., Díaz, J. A.,y Caicedo-Girón, J. Universidad, Responsabilidad y Humanismo. Universidad Católica de Colombia. Colección Nuevos Pensadores. (p.p. 8796).

Escobar-Picasso, E y Escobar-Cosme, A. (2010). Principales corrientes filosóficas en bioética. Boletín médico del hospital Infantil de México.vol.67, No.3. pp 196-203

García. A. (2010). Bioética y la Dignidad de la Persona. Disponible en línea: http://www. redalyc.org/articulo.oa?id=29014472005.[Consulta: 2015, Febrero,06].

Holguín, Y. (2019). Aproximación a la teoría de la Ética Convergente para la formación en bioética para investigación. Medicina y ética: Revista internacional de bioética, deontología y ética médica, 30(3), 701-722.

Islas, D. S. C., \& Cortés, F. V. (2020). Presencia de la bioética en la investigación de la Universidad Autónoma Metropolitana. Perfiles Educativos, 42(168). 
Junges, J. (2008). El Estatuto Epistemológico De La Bioética. UNISINOS. http://www.unesco.org.uy/shs/fileadmin/templates/shs/archivos/ TrabajosLibresBioetica/16.\%20E1\%20estatuto\%20espistemologico. pdf[[Consulta: 2016, Agosto, 06]

Maldonado, C. (2005). Acerca del Estatuto Epistemológico de la Bioética. Universidad Externado de Colombia. Derecho y Vida. Lus et vita. $N^{\circ}$ LII y LIII. http:// www.researchgate.net/publication/228300449_A_Reflection_about_the Epistemological_Status_of_Bioethics. ISSN1692-6455.

Maldonado, C y Gómez N. (2013) Ciencias de la complejidad desarrollo tecnológico y bioética ¿Para qué sirve la bioética global? Universidad del Rosario, Bogotá, Colombia. http://www.academia.edu/7850148/Ciencias_de_la_ complejidad_desarrollo_tecnológico_y_bioética

Maldonado, C. (2015) Complejidad de la Bioética Universidad. Del Rosario, Bogotá, Colombia. Disponible en línea: http://thelos.utem.cl/2015/12/complejidadde-la bioética/

Martin, V. (2014). Dilemas de la bioética en el siglo XXI. Revista lasallista de investigación - Vol. 11 No. 1 - 2014 - 56•62 Disponible en línea: www. scielo.org.co/pdf/rlsi/v11n1/v11n1a07.pdf [Consulta: 2018, Agosto, 10].

Martínez, M.(2008)¿Hacia una bioética global? A propósito de la propuesta de V.R. Potter Foro, Nueva época, núm. 8/2008: 327-341 ISSN: 1698-5583. https://revistas. ucm.es/index.php/FORO/article/viewFile/FORO0808220327A/13542

Martínez, M. (2011). Ciencia y Arte de la Metodología Cualitativa. Editorial Trillas. México.

Mazzanti, M. (2010). Investigación Transdisciplinaria en Bioética Moderadora de la Importación de Políticas Bioéticas Globales. Revista Colombiana de Bioética. Vol. 5(2). pág. 154-163. http://www.redalyc.org/articulo. oa?id=189218186015 
Pérez, R. (2008). Una Ética para el Hombre. Principios y Valores. http://catedraderamiro. blogspot.com/2008/03/bioetica.html

Rondón Y, Córdova V. y Rodríguez D. (2012). Hacia una nueva concepción de la gerencia en la educación superior venezolana. UDO. Revista Saber, Volumen 24(1), pág.: 83-89. http://ojs.udo.edu.ve/index.php/saber/article/ download $/ 405 / 288$

Schimidt, L. (2007) La Bioética en Venezuela, Primeras Huellas. Centro Universitario Sao Camilo.Vol.1(2).pág.:24-38. http://www.bioeticachile.cl/felaibe/ documentos/venezuela/Bioetica_Venezuela.pdf

Velasco J. (2009) Origen y desarrollo de una ciencia: la bioética. Univ. de Deusto. Facultad de Teología. http://hedatuz.euskomedia.org/7529/1/54147187.pdf 